\title{
MOLUSCOS, CRUSTÁCEOS Y EQUINODERMOS: RESTOS DE ORIGEN MARINO EN LOS YACIMIENTOS PREHISTÓRICOS
}

\author{
Molluscs, crustacean and echinoderms: marine remains in Prehistoric sites
}

\author{
Esteban ÁLVAREZ FERNÁNDEZ \\ Departamento de Prehistoria, $\mathrm{H}^{\mathrm{a}}$ Antigua y Arqueología \\ Universidad de Salamanca \\ E-mail: estebanalfer@hotmail.com
}

Fecha de recepción: 20-01-2010

Fecha de aceptación: 29-01-2010

RESUMEN: Se dan a conocer algunos de los resultados sobre las recientes investigaciones arqueozoológicas llevadas a cabo en diferentes yacimientos de la Región Cantábrica. Se presentan datos de diferentes restos marinos (moluscos, cangrejos, percebes, bellotas de mar y erizos) y se reflexiona sobre la información de diferente tipo que nos ofrecen (paleoeconómica, paleoclimática, etc.).

Palabras clave: explotación de recursos marinos, Prehistoria, Región Cantábrica

ABSTRACT: This paper presents some of the results of recent archaeozoological research carried out at a number of sites in Cantabrian Spain. It provides data about various types of marine remains (molluscs, crabs, goose barnacles, acorn barnacles and sea urchins) and reflects on the different kinds of information they provide (palaeo-economy, palaeoclimate, etc).

Keywords: marine resources exploitation, Prehistory, Cantabrian Spain 


\section{INTRODUCCIÓN}

El estudio de los recursos marinos documentados en los yacimientos prehistóricos de la Región Cantábrica posee una gran tradición. Su interés se remonta a finales del siglo XIX. Sin embargo, es a partir de mediados de los años sesenta del siglo pasado cuando se comienzan a utilizar en las excavaciones arqueológicas cribas para realizar el tamizado fino de los sedimentos, lo que posibilitó un mayor registro de restos de moluscos, pero también de otros recursos marinos (peces, crustáceos, equinodermos, etc.). Así, en los últimos años, se ha incrementado notablemente la información sobre este tipo de recursos en la Prehistoria.

En este artículo se presentan algunos de los resultados de nuestras investigaciones que llevamos haciendo desde hace algunos años en diferentes restos arqueoozológicos marinos, concretamente en moluscos, crustáceos y equinodermos.

\section{LOS RESTOS DE MOLUSCOS}

Los moluscos son los restos de origen marino más abundantes en los yacimientos arqueológicos. Las primeras evidencias de su explotación en la Región Cantábrica proceden de yacimientos adscritos a finales del Paleolítico medio (Álvarez-Fernández, 2005-2006) y su explotación intensiva tiene lugar a comienzos del Holoceno, durante el Mesolítico (Álvarez Fernández, e. p. 1).

La utilización de mallas metálicas finas en los yacimientos ha posibilitado la recogida de conchas completas y de fragmentos de pequeño tamaño. Algunas de estas piezas han sido transformadas con anterioridad en objetos de adorno-colgantes, como ocurre en el Magdaleniense de La Garma A, en el que se han documentado, entre otras especies, un ejemplar del gasterópodo de origen mediterráneo Homalopoma sanguineum (Linnaeus, 1758), posiblemente transformada en objeto de adorno (Álvarez Fernández, e. p. 2). (Figura 1). En otros yacimientos, como en el francés de Beg-an-Dorchern (campaña de 2001), se han recogido otras especies de pequeño tamaño, como Lacuna sp. y Bittium sp., procedentes de la superficie de rocas, y otras como Ansates pellucida (Linnaeus, 1758) y Striarca lactea (Linnaeus, 1758), que habitaban sobre algas (Dupont, 2006). 
La recogida de partes diagnósticas de conchas como son, por ejemplo, los ápices de diferentes especies de Patella sp. y de Osilinus lineatus (da Costa, 1778), nos han posibilitado incrementar del NR (Número de Restos) y del NMI (Número Mínimo de Individuos) total en el conchero mesolítico de Jaizkibel 3, donde se han empleado mallas metálicas de $2 \mathrm{~mm}$. de luz.

Otro ejemplo de recogida sistemática de restos malacológicos procede de las investigaciones llevadas a cabo en distintos concheros holocenos del $\mathrm{NE}$ de Asturias. Aquí, se han desmenuzado las concreciones calcáreas que engloban diferentes restos de origen animal, documentándose los primeros objetos de adorno-colgantes mesolíticos en este tipo de contextos. Así, en La Poza l'Egua, hemos identificado dos conchas marinas perforadas de las especies Littorina obtusata (Linnaeus, 1758) y Nassarius reticulatus (Linnaeus, 1758), además de otros restos de origen marino (vértebras de peces, púas y placas de erizos de mar, etc.) (Arias Cabal, P., et al., 2007).

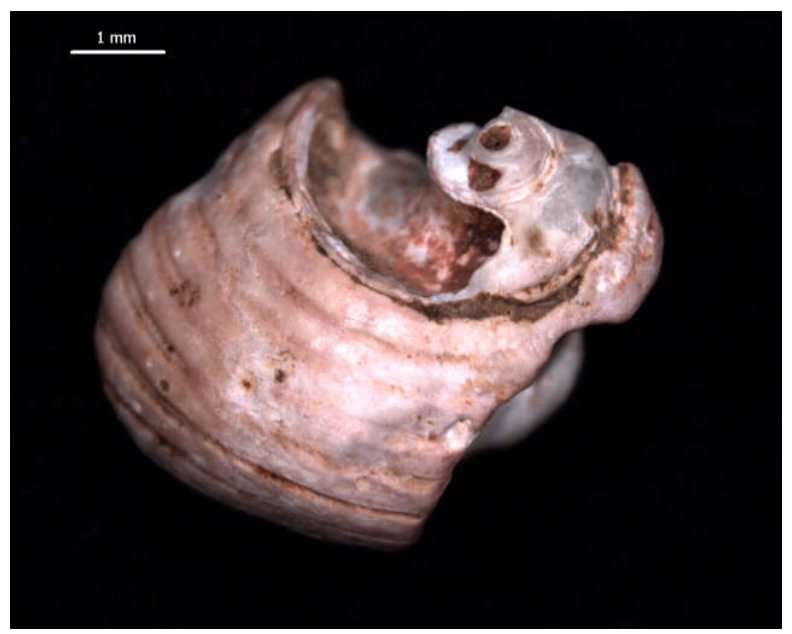

Figura 1. La Garma A (Omoño, Cantabria). Magdaleniense. Ejemplar de Homalopoma sanguineum.

\section{LOS CRUSTÁCEOS}

Los restos de cangrejos que hemos documentado en los yacimientos arqueológicos son las pinzas. Por sus características, estos restos son bastante 
fáciles de identificar en las excavaciones arqueológicas.

Los cangrejos que se documentan en los yacimientos cantábricos son variadas: Cancer pagurus (Linnaeus, 1758), Carcinus maenas (Linnaeus, 1758), Xantho sp., Eriphia verrucosa Heller 1863, Necora puber (Linnaeus, 1758) and Pachygrapsus marmoratus (Fabricius, 1787). Se encuentran en sustratos rocosos, entre las grietas y bajo las rocas, sobretodo en la zona intermareal (zona que queda cubierta durante las pleamares y descubierta durante las bajamares). De las especies citadas, C. maenas es la más abundante y ubicuista. Vive en sustratos rocosos, arenosos y fangosos, a lo largo de toda la zona intertidal. Todas las especies citadas son cosmopolitas y viven en aguas templadas y frías. Para su clasificación a nivel de especie es fundamental poseer una colección de referencia y realizar análisis biométricos. También es posible calcular, a partir de las pinzas arqueológicas, el tamaño y el peso del cangrejo e incluso el sexo del individuo (por ejemplo, en las especies C. pagarus (Linnaeus, 1758) y Uca tangeri (Eydoux, 1835). Por otra parte, ciertas especies nos dan datos de tipo paleoclimático, como E. spinifrons (Gruet, 2002; 2003; e.p.; Gruet \& Laporte, 1996).

Así, por ejemplo, en el Magdaleniense de Altamira ha podido ser calculado el NMI (2) a partir de NR (4) y ha podido reconstruirse la talla de los ejemplares de Xantho sp. y C. maenas. Las pinzas pertenecían a individuos adultos (el primero de 45/50 $\mathrm{mm}$ de longitud y el segundo $65 / 70 \mathrm{~mm}$ de longitud), lo que indica que estos cangrejos fueron recogidos intencionalmente por los grupos humanos. Además, la talla de los ejemplares arqueológicos sería más grande que los actuales debido probablemente a unas condiciones climáticas más frías que en la actualidad (Álvarez Fernández 2010).

En el yacimiento mesolítico francés de Beg-an-Dorchern Y. Gruet (e. p.) ha indicado que gran parte de los ejemplares de C. pagurus son de gran tamaño (140-150 mm). Puesto que estas tallas están presentes en los individuos que normalmente habitan la zona infralitoral (zona permanentemente cubierta por el mar), es posible que hayan sido recogidos en momentos de mareas vivas. Se ha documentado también un dedo de la especie Maja squinado (Herbst, 1788). Aunque esta especie es subtidal, pudo haber sido pescada en la zona intermareal a partir del mes de abril, periodo en el que esta especie migra desde la zona subtidal. No se descarta tampoco que esta especie haya 
sido pescada en pesquerías. La utilización de este tipo de estructuras antrópicas explicaría también la presencia de determinadas especies de peces en el yacimiento.

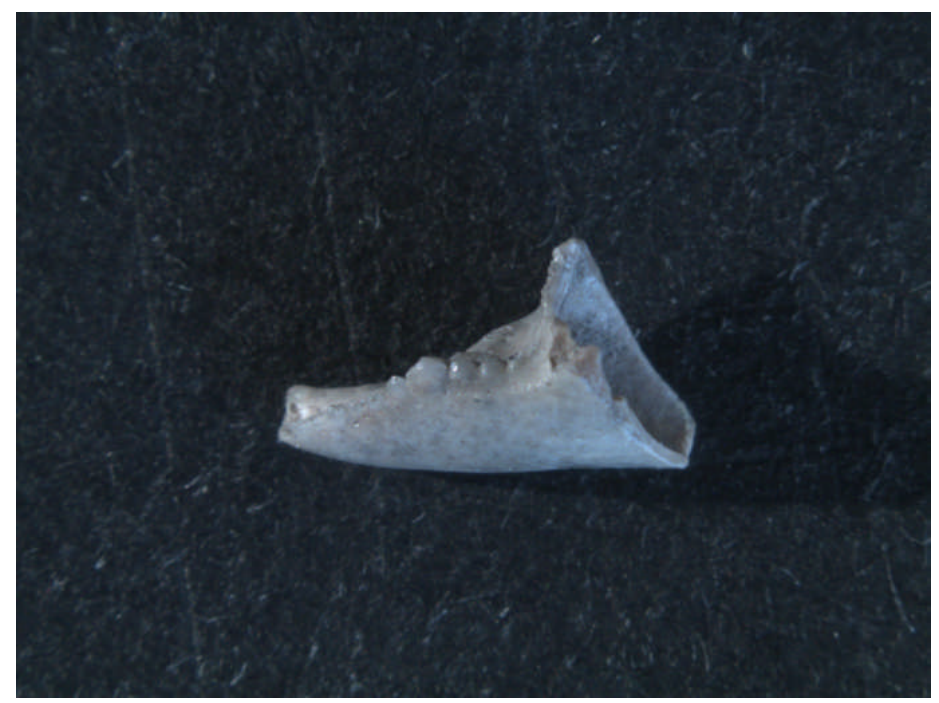

Figura 2. Los Gitanos de Montealegre (Sámano, Cantabria). Neolítico. Pinza de cangrejo de la especie Pachygrapsus marmoratus. Tamaño: $6 \mathrm{~mm}$.

En el Neolítico de Los Gitanos se han identificado las especies Xantho sp., E. verrucosa, N. puber and P. marmoratus. Junto a la presencia de individuos adultos, que muy posiblemente fueron consumidos por los grupos humanos, documentamos ejemplares de muy pequeño tamaño, que tal vez han llegado al yacimiento de forma indirecta (algas, etc.).

También se han documentado restos de percebes. Pollicipes pollicipes (Gmelin, 1790) ha sido explotado a partir del Holoceno, tal como se ha documentado en numerosos yacimientos del SW de Europa. Con anterioridad esta especie no existía en el Cantábrico, ya que no tolera las aguas frías. Así, su límite actual está en la ciudad de Brest, en la Bretaña francesa (Álvarez Fernández, et al. 2010).

Los percebes crecen en colonias, formando grupos o piñas sobre las rocas batidas por el oleaje en la zona intermareal (zona que queda cubierta durante las pleamares y descubierta durante las bajamares) e infralitoral (zona 
permanentemente cubierta). Los racimos se recogen de forma artesanal cuando la marea baja, momento en el que quedan al descubierto sobre las rocas. En los percebes se pueden distinguir dos partes: el pedúnculo o pie (parte inferior), la parte comestible del animal, y el capítulo (parte superior), formado por una especie de caparazón que está protegido por unas 40 placas calcáreas, de color blanco-nacarado a grisáceo y de diferente tamaño. Estas placas lo protegen del ataque de los depredadores y de la desecación en los momentos de bajamar. Esta parte se conoce vulgarmente como uña del percebe (Figura 3 izquierda)
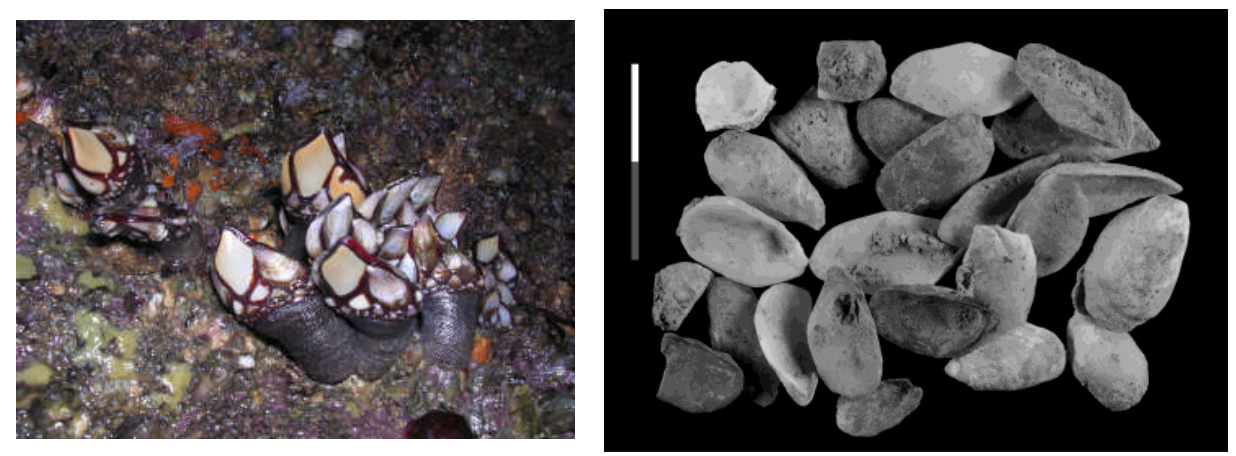

Figura 3. Izquierda. Ejemplares de percebe actuales (Foto: Y. Gruet). Derecha: Los Gitanos de Montealegre (Sámano, Cantabria). Neolítico. Placas de Pollicipes pollicipes. (Foto: L. Teira)

Las placas de mayor tamaño (tergum, scutum y carina) son las que comúnmente aparecen en los yacimientos arqueológicos de la Región Cantábrica. Su forma hace posible que puedan ser orientadas y lateralizadas, pudiéndose calcular el NMI a partir del NR. Así, en los niveles neolíticos de Los Gitanos de Montealegre (Sámano, Cantabria) se han podido contabilizar 77 individuos a partir de 288 placas (Figura 3 derecha). La captura de estos crustáceos en zonas muy batidas por el mar implicaría el conocimiento y uso de técnicas de captura específicas.

Por último, hemos documentado balanos de diferentes especies en los yacimientos prehistóricos de la Región Cantábrica

Los balanos son crustáceos cirrípedos que habitan sobre otros animales marinos, sobre las rocas, barcos y objetos flotantes. Poseen una forma 
cónica formada por seis placas y se fijan a ellos por su base, a veces calcárea. Son conocidos como bellotas de mar.

En algunas regiones del mundo son consumidos como alimento, como el balano de grandes dimensiones Megabalanus azoricus (Pilsbry, 1916) (Dionísio, M. A., et al., 2007). Sin embargo, los ejemplares que documentamos en los yacimientos de la Región Cantábrica son de muy pequeño tamaño y seguramente vinieron adsosados a la superficie externa de diferentes especies de gasterópodos y bivalvos, como lapas y mejillones.

Pero, aunque carecen de valor alimenticio, estos crustáceos nos dan datos de tipo paleoclimático y acerca del lugar de la costa (zonas más o menos expuestas) en el que han sido recolectadas las diferentes especies de moluscos sobre las que habitan. Así, por ejemplo, la clasificación de especies boreales Semibalanus balanoides (Linnaeus, 1767) en el Magdaleniense inferior de Altamira y en el Magdaleniense medio de La Garma A y de Balanus crenatus (Brugière, 1789) en el ultimo yacimiento citado, indicarían que la temperatura del agua del mar sería más fría que en la actualidad y que los moluscos sobre los que vivían (Patella sp.) fueron recogidos en zonas muy batidas por el mar (Álvarez Fernández, 2009; i. p. 3) (Figura 4). En los yacimientos mesolíticos franceses de Beg-er-Vil y Beg-an-Dochenn la presencia masiva de la especie de aguas cálidas Balanus perforatus (Brugière, 1789) indicaría que los moluscos y otros organismos sobre los que han sido documentados proceden de la zona infralitoral, es decir de una zona muy batida por el mar (Dupont et Gruet 2005).
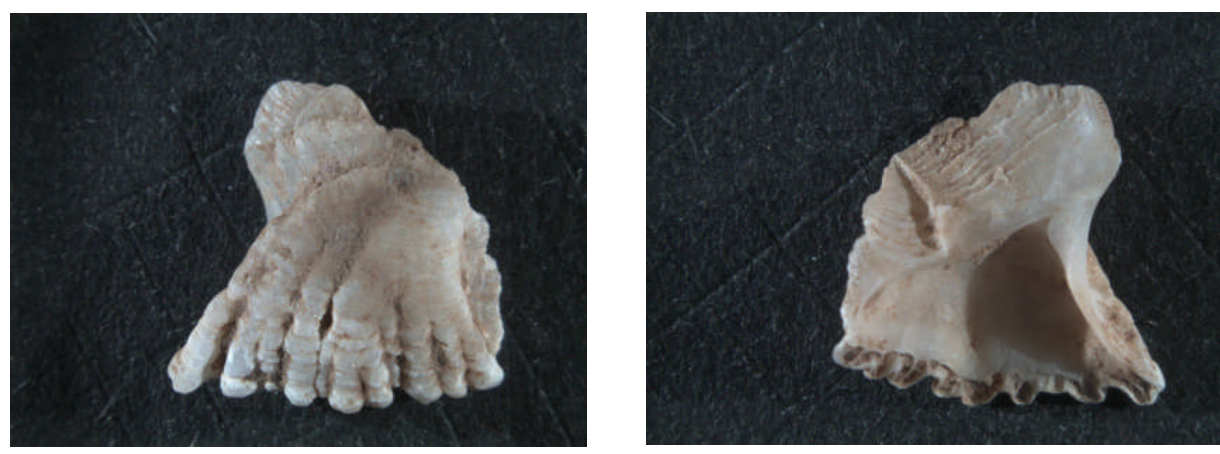

Figura 4. La Garma A (Omoño, Cantabria). Magdaleniense. Placa de balano de la especie Semibalanus balanoides. Tamaño: 7,5 mm. 
Particularmente es interesante y único el resto de balano documentado en los niveles de Magdaleniense medio de Las Caldas. Pertenece a Coronula diadema (Linnaeus, 1758), especie parásita que vive exclusivamente sobre las ballenas, en cuya piel se enraíza. Su presencia en el yacimiento es una prueba del posible acarreo de trozos de carne de cetáceo con piel, ya que no es posible desprender el cirrípedo sin cortar dicha carne (Corchón et al., 2008) (Figura 5)

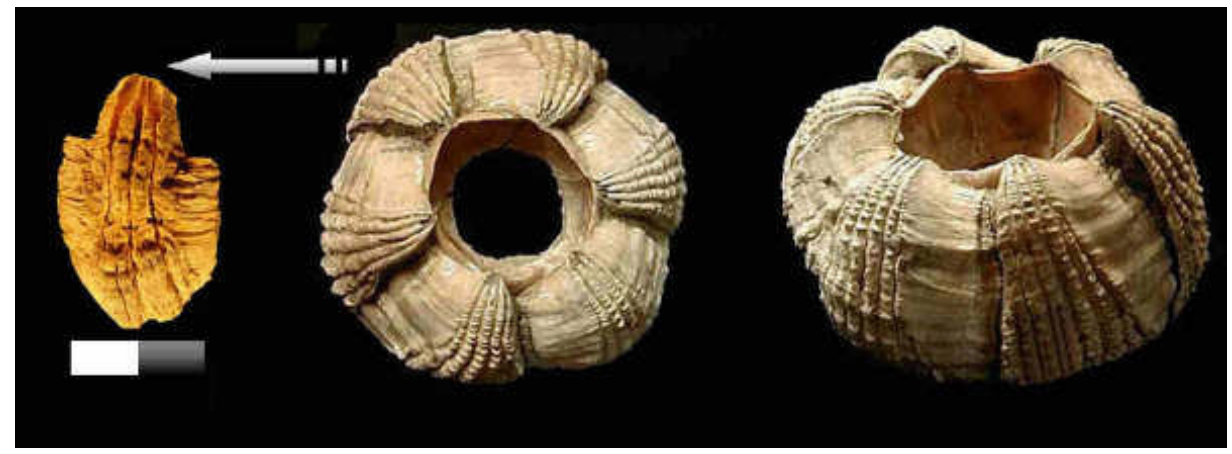

Figura 5. Izquierda: Las Caldas (Oviedo, Asturias). Magdaleniense. Placa de balano de la especie Coronula diadema.; Derecha: Ejemplar actual de la misma especie. Foto: S. Corchón.

\section{LOS EQUINODERMOS}

Los erizos de mar aparecen frecuentemente en los yacimientos arqueológicos desde el Magdaleniense. Son bastante abundantes en los concheros holocenos de la Región Cantábrica, en los que son fáciles de identificar fragmentos de caparazón y púas.

Hasta el momento, la única especie que hemos documentado en los yacimientos de la Región Cantábrica es Paracentrotus lividus (Lamarck, 1816) o erizo de mar común, la especie de erizo más frecuente en esta región. El diámetro del caparazón de Paracentrotus lividus oscila entre 4 y 7 cm., aunque existen ejemplares que pueden llegar a los $10 \mathrm{~cm}$. Las púas de un individuo medio son de unos $3 \mathrm{~cm}$ de longitud. Su cuerpo posee un contorno circular ligeramente deprimido. Su coloración es púrpura, casi negra y las espinas pueden ser de color violeta, verde oliva, rojizo y marrón. Vive sobre la pla- 
taforma continental (-35/-40 m), no alcanzando profundidades superiores a los $80 \mathrm{~m}$. Coloniza las rocas, excavando cavidades, pero también vive sobre las praderas de Posidonia y sobre fondos arenosos o coralígenos de superficie sin pendiente (Campbell, 2008; Gruet, 2003).

Esta especie ha sido reconocida en sitios como la Garma A (Magdaleniense) o Los Gitanos de Montealegre (Figura 6). En La Garma A, además de placas y púas, hemos documentado otras partes anatómicas (placas hemipiramidales, rótulas, aurículas) que nos posibilitarán identificar las especies recogidas y calcular el NMI, tal como se ha hecho a otros yacimientos como el francés de Bahía de Lannion, de época romana (Campbell, 2008). En los niveles neolíticos y calcolítico de Los Gitanos su clasificación a nivel de especie se ha realizado por la distribución de los tubérculos de las placas y las estriaciones y protuberancias en forma de alubia de las púas. La ausencia de partes anatómicas características y la extrema fragmentación de las placas impide hacer el cálculo del NMI. Esto, unido a la escasez de restos por nivel arqueológico nos hace dudar de su recogida directa por parte de los grupos humanos en el mar. Sería más probable que estos restos formasen parte del alimento de las especies ictológicas capturadas en la costa y transportadas a la cueva.

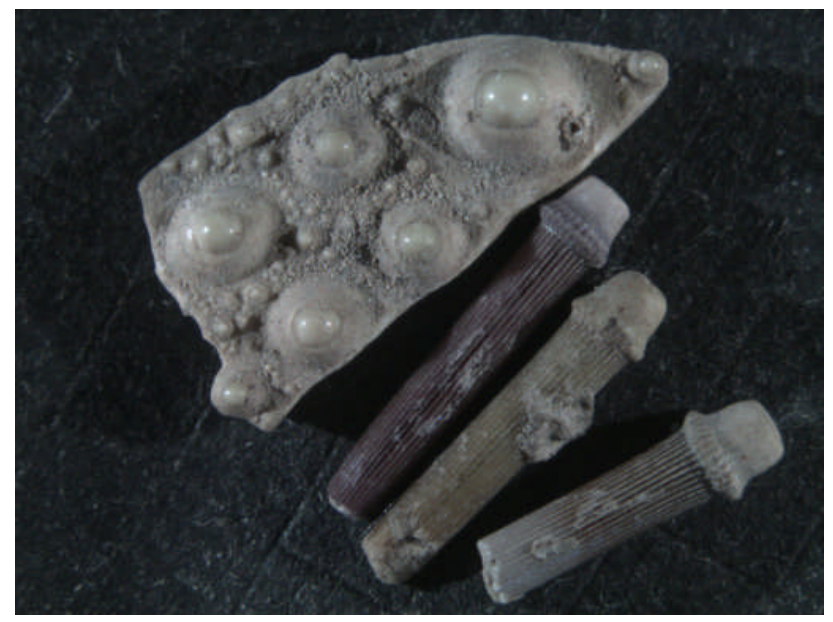

Figura 6. Los Gitanos de Montealegre (Sámano, Cantabria). Neolítico. Placas y púas de la especie Paracentrotus lividus. Tamaño: $15 \mathrm{~mm}$ 


\section{ALGUNAS RELEXIONES}

Los restos de origen marino que ha gozado de una atención mayor a partir de los años sesenta del siglo pasado han sido los moluscos con interés bromatológico (caracolillos, lapas, ostras, mejillones, etc.). Estos están presentes en un número importante de yacimientos cantábricos. El empleo de cribas para tamizar el sedimento ha posibilitado el incremento, tanto en el NR, como en el NMI. Sin embargo, en las cribas también han aparecido otros restos arqueozoológicos de origen marino a los que prácticamente no se les ha prestado atención hasta la actualidad y que nos acercan igualmente al conocimiento de las relaciones de los grupos humanos y las zonas costeras en la Prehistoria.

La presencia en los sitios de determinadas especies de moluscos de muy pequeño tamaño puede indicarnos la preferencia por parte de los grupos humanos, de recoger en las playas conchas de especies que no poseen valor alimenticio con el objeto de transformarlas en colgantes. Algunas de ellas, de clara procedencia mediterránea, nos hablarían de redes de contactos a larga distancia, al menos, ya desde comienzos del Paleolítico superior (Álvarez Fernández, 2006). Otras especies se introducirían de forma involuntaria en el yacimiento y podrían haber ido adheridas a algas, rocas o arena, recogidas en el entorno marino.

Además de restos de moluscos, también se han documentado restos de crustáceos y de equinodermos. Una primera valoración de su presencia en los yacimientos mesolíticos y neolíticos indica que los cangrejos, los percebes y los erizos de mar no eran muy abundantes. Estos animales no parecen haber sido demasiado importantes en la dieta de los grupos de los últimos cazadores-recolectores y primeros productores de alimentos. En la Región Cantábrica su recogida sería oportunista y se llevaría a cabo durante la recolección de diferentes especies de moluscos marinos (fundamentalmente $\mathrm{Pa}$ tella sp. y $O$. lineatus), animales que sí son importantes en la dieta de estos grupos (Álvarez Fernández, e. p.1). La presencia de ciertos restos de crustáceos, sin embargo, se puede deber a la acción indirecta del hombre. Así, al igual que ocurre con algunos restos de moluscos, los restos de cangrejos de pequeño tamaño podrían venir con algas y los de erizo, en los intestinos de diferentes especies de peces acarreados a las cuevas y abrigos. En cuanto a los balanos, los de pequeño tamaño que habitan sobre las lapas 
y otros moluscos, seguramente fueron traídos al yacimiento de forma involuntaria. El balano de ballena, además, pudo haber sido acarreado con trozos de carne procedentes de un animal varado en la playa.

Finalmente, hay que indicar que los restos de diferentes especies de moluscos, crustáceos y equinodermos pueden ser indicadores de cambios climáticos y pueden darnos información de la zona de la costa donde eran recogidos. Así, por una parte, nos pueden hablar de unas condiciones climáticas mucho más frías que en la actualidad, tal como ocurre con la presencia de especies de balanos como Semibalanus balanoides durante el Magdaleniense. Otras, como Pollicipes pollicipes, nos informan de la existencia de unas condiciones climáticas más cálidas. Por otra parte, la presencia de la especie citada de bellota de mar nos indicaría que los moluscos sobre los que vivía fueron recogidos por los grupos humanos en zonas muy batidas por el mar y que recolectaban recursos marinos de la zona intermareal, al menos, desde el Magdaleniense inferior.

\section{BIBLIOGRAFÍA}

Álvarez-Fernández, E., 2005-2006. La explotación y utilización de los moluscos marinos durante el Paleolítico superior y el Mesolítico en la Cornisa Cantábrica y en el Valle del Ebro: pasado y presente de la investigación. En: Homenaje al Prof. Jesús Altuna. Vol. I. Sociedad de Ciencias Aranzadi (Munibe 57), San Sebastián: 359-368.

Álvarez Fernández, E. 2006. Los objetos de adorno-colgantes del Paleolitico superiory del Mesolitico en la Cornisa Cantábrica y en el Valle del Ebro: una visión europea. Salamanca: Ed. Universidad de Salamanca (Colección Vítor no 195). Salamanca.

Álvarez Fernández, E. 2009. Análisis arqueomalacológico de la cueva de Altamira (Santillana del Mar, Cantabria): excavaciones de J. González Echagaray y L. G. Freeman. Complutum 20 (1): 55-70.

Álvarez-Fernández, E. e. p. 1. Humans and marine resource interaction reappraised: archaeofauna remains during the Late Pleistocene and Holocene in Cantabrian Spain.

Álvarez Fernández, E. e. p. 2. Las conchas marinas como evidencias 
de contactos a larga distancia de los grupos de cazadores-recolectores en la Península Ibérica. In: Gentes del Mar. Historia y Arqueología en el litoral del Arco Atlántico.

Álvarez-Fernández, E. e. p. 3. Limpets \& Periwinkles in Cantabrian Spain between 22,000 and 15,000 cal BC: Archaeomalacological Remains at Altamira Cave.

Álvarez-Fernández, E.; Ontañón, R. \& Molares, J., 2010. Archaeological Data on the Exploitation of the Gooseneck Barnacle Pollicipes pollicipes (Gmelin, 1790) in Europe. Journal of Archaeological Science 37 (2): 402-408.

Arias Cabal, P.; Fernández-Tresguerres Velasco, J. A.; Álvarez Fernández, E.; Armendariz Gutiérrez, A.; Cueto Rapado, M.; Fano Martínez, M. A.; Fernández García, R.; Garralda Benajes, M. D.; Mensua Calzado, C. \& Teira Mayolini, L. C. 2007. Excavación arqueológica de urgencia en la cueva de La Poza l'Egua (Lledías, Llanes), Excavaciones Arqueológicas en Asturias, V. Principado de Asturias, Oviedo: 227-239.

Campbell, G., 2008. Sorry, Wrong Phylum: A Neophyte Archaeomalacologist's Experiences in Analyzing a European Atlantic Sea Urchin Assemblage. Archaeofauna 17: 77-90.

Dionísio M. A.; Rodrigues, A. \& Costa, A. C. 2007. Reproductive cycle of Megabalanus azoricus (Pilsbry, 1916), the Azorean barnacle”. Invertebrate Reproduction and Development 50(3): 155-162.

Dupont, C. 2006. La malacofaune des sites mésolithiques et néolithiques de la façade atlantique de la France. Contribution à l'economie et à l'identité culturelle des groupes concernés. B. A. R. International Series 1571. Oxford.

Dupont, C. \& Gruet, Y., 2005. Malacofaune et crustacés marins des amas coquillieres mésolithiques de Beg-an-Dorchenn (Plomeuer, Finistèrre) et de Berg-er-Vil (Quiberon, Morbihan). In: Marchand, G., Tresset, A. (Eds.): Unité et diversité des processus de néolithisation sur la façade atlantique de l'Europe (6e-4e millénaires avant J.-C.). Table ronde de Nantes (Nantes, 2627 avril 2002). Mémoire XXXVI de la Société Préhistorique Française. Paris: 139-161.

Corchón, M. S.; Mateos, A.; Álvarez-Fernández, E.; Delclòs, X.; Pe- 
ñalver, E. \& van der Made, J., 2008. Ressources complémentaires et mobilité dans le Magdalénien Cantabrique. Nouvelles données sur les mammifères marins, les crustacés, les mollusques et les roches organogènes de la Grotte de Las Caldas (Asturies, Espagne). L'Anthropologie 112 (2): 284-327.

Gruet, Y., 2002. Reconnaissance de quelques espèces communes de crustacés (balanes et crabes: application au site Mésolithique de Beg-er-Vil (Morbihan, France). Revue d'Archéometrie 26: 125-139.

Gruet, Y., 2003. Les Crustacés et les Echinodermes. In: Dupont, C., Gruet, Y., Leroy, A., Marchand, G., Pailler, Y. \& Sparfel, Y. (Eds.): Le site préhistorique de Béniguet-3 (île Béniguet, Le Conquet, Finistère). Bulletin de l'AMARAI 16: 14-18.

Gruet, Y. (e.p.): Les crustacés (crabes et cirripèdes). In: Dupont, C. et al. (Eds.). Beg-an-Dorchenn: une fenêtre ouverte sur l'exploitation du littoral par les peuples mésolithiques du sixième millénaire dans l'ouest de la France.

Gruet, Y., Laporte, L., 1996. Crabes pêchés au Néolithique Final à Ponthezières (Saint-Georges d'Oléron, Charente-Maritime): identifications, modes de pêche et application de la métrique. Actes du Colloque de Périgueux 1995. Supplément à la Revue d'Archéométrie, 197-201. 